\title{
Galenus-von-Pergamon-Preis 2012 - Die Jury hat entschieden
}

\begin{abstract}
Für manche ist er der inoffizielle Nobelpreis für Pharmakologie: der Galenus-von-Pergamon-Preis. Mitte Oktober wurden die drei diesjährigen Medaillen für exzellente Grundlagenforschung und herausragende Innovationen verliehen. Bei einer Gala in München nahmen Novartis, Roche und Dr. Thomas Worzfeld ihre Preise entgegen.
\end{abstract}

In der Kategorie „Primary Care“ wurde Fingolimod (Gilenya $\left.{ }^{\star}\right)$ von Novartis Pharma, in der Kategorie „Specialist Care“ Vemurafenib (Zelboraf ${ }^{\circledast}$ ) von Roche Pharma ausgezeichnet. In der Kategorie „Grundlagenforschung“" wurde das Team um Dr. Thomas Worzfeld, Bad Nauheim, für die Entwicklung eines neuen Ansatzes zur Therapie bei metastasierendem Brustkrebs geehrt. Anders als im Vorjahr hat sich die Jury sehr schwer getan wie ihr Präsident Professor Erland Erdmann, Köln, sagte: „Alle diesjährigen Kandidaten sind Innovationen. "Wie wichtig Innovationen seien, betonte Harm van Maanen, Executive Vice President Springer Medizin: „Innovationen sind unter den wichtigsten Gütern, die wir haben.“ Deshalb sollten sie gefördert werden.

\section{Neue Option für MS-Patienten}

Fingolimod ist ein orales Medikament zur Therapie von Patienten mit Multipler Sklerose (MS). Es ist zugelassen für bisher nicht behandelte Patienten, die an einer rasch fortschreitenden, schwer schubförmigen MS erkrankt sind. Zudem ist das Präparat indiziert zur Eskalationstherapie, wenn trotz Behandlung mit einem Beta-Interferon eine hohe Krankheitsaktivität vorliegt. Fingolimod verringerte die Schubrate im Vergleich zu Interferon in einer Zulassungsstudie um 52 \% (0,33 vs. 0,16 Schübe/Jahr).
Vemurafenib ist die erste Option für eine personalisierte Therapie bei inoperablem oder metastasiertem Melanom. Das Medikament in Form von Filmtabletten ist für Patienten zugelassen, deren Tumoren ein mutiertes BRAF-Gen aufweisen. In der Zulassungsstudie betrug die geschätzte mediane progressionsfreie Überlebenszeit 5,3 Monate - unter Standardchemotherapie mit Dacarbazin sind es 1,6 Monaten. In der VemurafenibGruppe war zudem das Sterberisiko um $63 \%$ und das Progressionsrisiko um $74 \%$ verringert. Die erste Entwicklungsphase hatte das US-Unternehmen Plexxikon bestritten.

Worzfeld und sein Team haben herausgefunden, dass der Rezeptor Plexin-B1 eine besondere Bedeutung für die Metastasierung bei Brustkrebs hat. Anhand von Gewebeproben von Patientinnen mit Mammakarzinom stellten sie fest, dass die Überlebenschance sich besserte, je weniger Plexin-B1 im Tumorgewebe vorhanden war. Inzwischen steht ein monoklonaler Antikörper zur Verfügung, der derzeit präklinisch getestet wird. „Anfangs dachten wir nicht, dass wir mit unserer Forschung bei Brustkrebs landen würden“, so Worzfeld, bei der Preisverleihung in München - Ausgangspunkt waren neurowissenschaftliche Fragen. Geplant sind Untersuchungen mit dem Antikörper auch beim Magen- und beim Ovarialkarzinom.

Peter Leiner

\section{Springer Medizin CharityAward 2012}

\section{Balthasar Kinder- und Jugendhospiz geehrt}

Auch der Springer Medizin CharityAward 2012 wurde im Rahmen der Gala verliehen. Mit der Auszeichnung, die Gesundheitsminister Daniel Bahr überreichte, wird die Arbeit des „Balthasar Kinder- und Jugendhospizes“ in Olpe geehrt. Die Einrichtung in Trägerschaft der Gemeinnützigen Gesellschaft der Franziskanerinnen zu Olpe wurde 1998 gegründet und ist das erste Kinder-und Jugendhospiz in Deutschland. Die Unterhaltung einer solchen Einrichtung birgt immer wirtschaftliche Risiken, somit ist das Haus stark von Spenden abhängig.

Der CharityAward umfasst einen Barscheck über $50.000 €$ und ein Medienpaket über weitere $100.000 €$. Es beinhaltet individuelle Dienstleistungen für den Gewinner: Springer Medizin unterstützt den Gewinner gezielt bei seiner zukünftigen Öffentlichkeitsarbeit. „Wir sind stolz und glücklich,” freute sich Hospizleiter Rüdiger Barth. „Durch das Medienpaket können wir unmittelbar Spenden generieren und zugleich auch neue Kontakte knüpfen, die vielleicht in eine langfristige Unterstützung münden. Eine doppelte Hilfe."

Außerdem vergeben wurde der mit $5.000 €$ dotierte Mutmacher-Preis an „Hilfe für Kinder krebskranker Eltern".

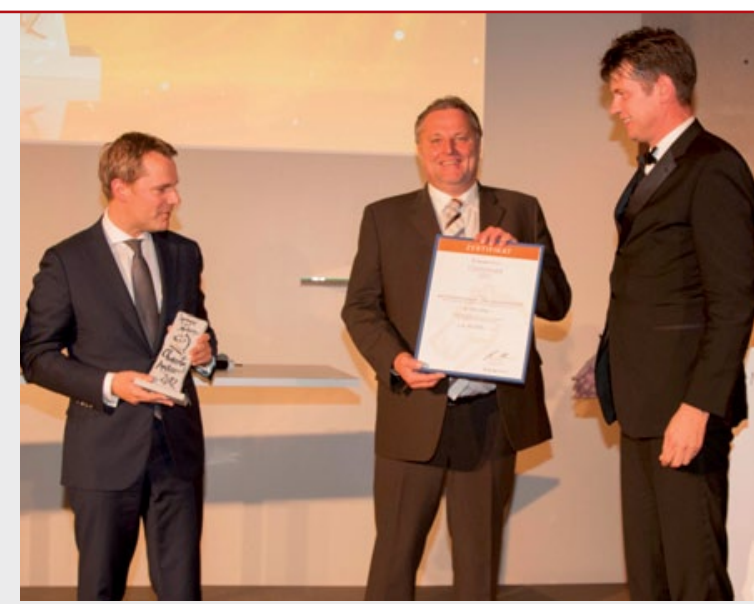

Bundesgesundheitsminister Daniel Bahr und Springer-Medizin-Chef Harm van Maanen übergeben den CharityAward an Rüdiger Barth, Kinder- und Jugendhospiz in Olpe 